\title{
Utilization of Antenatal Care Services in Bangladesh: A Cross-sectional Study Exploring the Associated Factors
}

\author{
Ashis Talukder, (1) Tanjim Siddiquee, (1) Nabila Noshin, (1) Meher Afroz, \\ Benojir Ahammed, (ㄱ) Henry Ratul Halder
}

Statistics Discipline, Khulna University, Khulna, Bangladesh

\section{ABSTRACT}

Objectives: The study aims to identify the associated factors with antenatal care (ANC) service utilization by pregnant women in Bangladesh.

Methods: Using Bangladesh Demographic and Health Survey, 2014, Chi-square association test detected significant explanatory variables for the number of ANC visits. For adjusted effects estimation, the study considered multinomial logistic regression (MLR) with relative risk ratio (RRR) and proportional odds model (POM). Akaike Information Criterion (AIC) compared and selected a better-fitted model.

Results: This study included 4488 responses who are women aged from 15-49 years. From MLR, primary educated women ( $R R R=1.344,95 \% \mathrm{Cl}=1.253-1.747, \mathrm{p}<0.001)$ with highly educated partners $(\mathrm{RRR}=1.478,95 \%$ $\mathrm{Cl}=1.255-1.908, \mathrm{p}<0.001$ ) were more likely to use ANC services. Besides, women belonging to the middle ( $R R R=1.209,95 \% \mathrm{Cl}=1.114-1.644, \mathrm{p}<0.001)$ and rich ( $R R R=1.506,95 \% \mathrm{Cl}=1.307-1.970, \mathrm{p}<0.001)$ wealth index had more chances of utilizing ANC services. However, ANC attendance decreased significantly with the increment in birth order $(p<0.001)$. ANC visits was also lower for rural women ( $R R R=0.711,95 \% \mathrm{Cl}=0.555$ 0.891, $\mathrm{p}<0.001$ ), Dhaka ( $R R R=0.623,95 \% \mathrm{Cl}=0.493-0.955, \mathrm{p}<0.001)$, Khulna ( $R R R=0.457,95 \% \mathrm{Cl}=0.388-0.687$, $\mathrm{p}<0.001$ ), and Rangpur ( $R R R=0.579,95 \% \mathrm{Cl}=0.484-0.780, \mathrm{p}<0.001)$ divisions.

Conclusion: To increase the ANC attendance, improvement of maternal education in rural areas is highly recommended. Dhaka, Khulna, and Rangpur divisions also need special focus.

Keywords: Antenatal care, Bangladesh, birth order, maternal age

\section{INTRODUCTION}

Maternal mortality is one of the strongest challenges to human development worldwide. Almost $99 \%$ of the maternal deaths occur in developing countries, mainly as a result of the disgraceful failure of healthcare schemes. ${ }^{[1]}$ According to the World Health Organization (WHO), a half-million women died in 2005 due to pregnancy-related complications. ${ }^{[2]}$ Besides, nearly $80 \%$ of global maternal mortality is directly related to pregnancy ${ }^{[3]}$ These unwanted deaths can be prevented with major health interventions, such as proper utilization of antenatal care (ANC). ${ }^{[4,5]}$

CCopyright 2021 by Anatolian Journal of Family Medicine Available online at Www.anatoljfm.org OPEN ACCESS

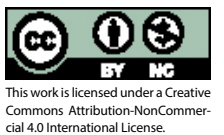

ANC is the most essential care given to pregnant women for their safe pregnancy and sound child. ${ }^{[6]}$ This should be administered at the early stages of pregnancy for rapid diagnosis of pregnancy-related complications. ${ }^{[6]} \mathrm{ANC}$ is a crucial element that can provide pregnant women with a comprehensive range of health promotion and preventive healthcare services. ${ }^{[7]}$ Women can enter ANC services either by visiting a healthcare center (where these services are 
available) or from trained medical personnel (such as doctor, nurse, midwife, medical officer, etc.). One of the most significant aspects of ANC is to provide health information and services that improve the health of both women and their children. ANC can also support pregnant women by addressing risk signs and symptoms that help early collaboration with a healthcare provider. ${ }^{[8]}$

During the first ANC visit, an ANC card is given to the pregnant woman with the first record of her pregnancy. The first visit to the ANC is also important since during this time a woman has a complete health checkup for pregnancy complications and gestational age..$^{[9]}$ After the first visit, the woman is thought to be seeking future ANC visits to detect possible risks (such as preterm delivery and adequately handle these complications, etc.). ${ }^{[9]}$ Recently, the WHO recommended that women should receive their first visit within the first 12 weeks of gestation, with subsequent visits at $20,26,30,34,36,38$, and 40 weeks. ${ }^{[10]}$ Moreover, ANC along with emergency obstetric and skilled delivery care is a crucial component of healthcare services that minimizes maternal mortality and improving newborn health. ${ }^{[1]]}$

The Bangladesh Demographic and Health Survey (BDHS) reported that ANC visits from any healthcare facilities increased by $17 \%$ between 2004 and 2007 . The increasing trend is also visible in the course of recent years (from $68 \%$ in 2011 to $74 \%$ in 2014). ${ }^{[12]}$ However, ANC attendance from therapeutically prepared facilities over the same period expanded only by $7 \%$ (from $51 \%$ to $55 \%$ ). ${ }^{[13]}$ The accessible statistic shows that about 12.000 women in Bangladesh are facing pregnancy-related complications. ${ }^{[13]}$ Early detection of high-risk pregnancies through ANC services and ensuring rapid access during an emergency to referral healthcare facilities can help to reduce maternal mortality. ${ }^{[14]}$ Several studies for Bangladesh found that an inadequate number of ANC visits (i.e. $\leq 4$ visits) have adverse outcomes on maternal and child health. ${ }^{[15-18]}$ Therefore, Bangladeshi healthcare professionals need to better understand the influential factors that affect the proper use of ANC services. For the use of healthcare services, there could also be large differences among women living in various socioeconomic groups. However, no prior studies applied both multinomial and ordinal regression models. Hence, the study aims to investigate affecting factors related to ANC visits for pregnant women by applying both multinomial and ordinal regression models for a nationally representative sample survey of Bangladesh.

\section{METHOD}

This study used secondary data collected by the National Institute of Population Research and Training and Demographic and Health Surveys (DHS). The sampling frame used for BDHS 2014 was the complete list of enumeration areas (EAs) covering the entire country prepared by the Bangladesh Bu- reau of Statistics. An EA is a geographic area that covers an average of 113 households. The sampling frame contains information about the EA location, type of residence (urban or rural), and the estimated number of residential households. A sketch map that delineates the EA geographic boundaries is available for each EA. Administratively, Bangladesh is divided into seven divisions. Each division is further subdivided into progressively smaller districts (zillas), subdistricts (thanas), unions, wards, and villages. An EA is either a village or a group of small villages, or a part of a large village. These divisions allow the whole country to be easily separated into small geographical areas with an urban-rural designation. The urban areas were further classified into two groups, city corporations and other than city corporations. The division size varies from 5.6\% (Sylhet) to 33.7\% (Dhaka). In Bangladesh, $23.3 \%$ of the households are in urban areas, $8.2 \%$ are in city corporations, and $15.1 \%$ are in other than corporations. ${ }^{[12]}$ Among 17989 selected households, interviews were performed in $98 \%$ of all chosen households. This only study considered married women of age 15-49 years who had at least one child in the last five years preceding the survey was conducted. The study population for this analysis was 4488 out of 17863 households. More details about the sampling procedure are available in the final report of BDHS 2014..$^{[12]}$

Respondents of the study were women of reproductive age who had ANC visits during pregnancy. Therefore, the number of ANC visits was the response variable. Briefly, the response variable was categorized into three groups such as no visit, 1-3 visits, and $\geq 4$ visits. This categorization provided precise information on the pattern of ANC service utilization that varies according to the risk factors. ${ }^{[19]} \mathrm{A}$ set of socioeconomic and demographic risk factors associated with ANC visit were considered as explanatory variables to fit both multinomial and ordinal regression models. Explanatory variables included women's education (illiterate, primary, secondary, higher), partner's education (illiterate, primary, secondary, higher), place of residence (urban, rural), wealth index (categorized based on terciles), maternal age at birth ( $<20$ years, 20-34 years, 35-49 years), birth $\operatorname{order}\left(1^{\text {st }}, 2^{\text {nd }}, 3^{\text {rd }}\right.$ and $4^{\text {th }}$ or more), religion (Islam, others), and division (Barisal, Chittagong, Dhaka, Khulna, Rajshahi, Rangpur, Sylhet). Prior studies also found a significant association of these explanatory variables with ANC visits. ${ }^{[19,20]}$

All data analyses were performed using SPSS Version 23 (SPSS Inc., Chicago, USA) and R Version 3.5.2 (Bell Laboratories, New Jersey, USA). Baseline information was presented using descriptive statistical methods such as percentages and frequencies. The association between selected explanatory variables and ANC visit was examined by bivariate and multivariate analyses. In multivariate setup, multinomial logistic regression (MLR) and proportional odds model (POM) estimated the adjusted effects of explanatory variables on the ANC visit of pregnant women. For model selection, the Akaike Information Criterion (AIC) was used and selected a 
better-performed model with a minimum value of AIC.

\section{RESULTS}

This study included 4488 responses for pregnant women aged between 15 and 49 years. The result showed that 335 (53.6\%) of illiterate women never received ANC services, while 176 (52.4\%) of highly educated women availed ANC services at least four times. Partner's education also showed a similar result, for example, 477 (46.4\%) of women with illiterate partners received no ANC services and 123 (43.9\%) of women with highly educated partners received at least four ANC visits. Urban pregnant women receive more ANC services than their rural counterparts. The percentage of visiting at least four times was 357 (31.3\%) and 424 (15.4\%)

Table 1. The association between selected explanatory variables and attendance of antenatal care services

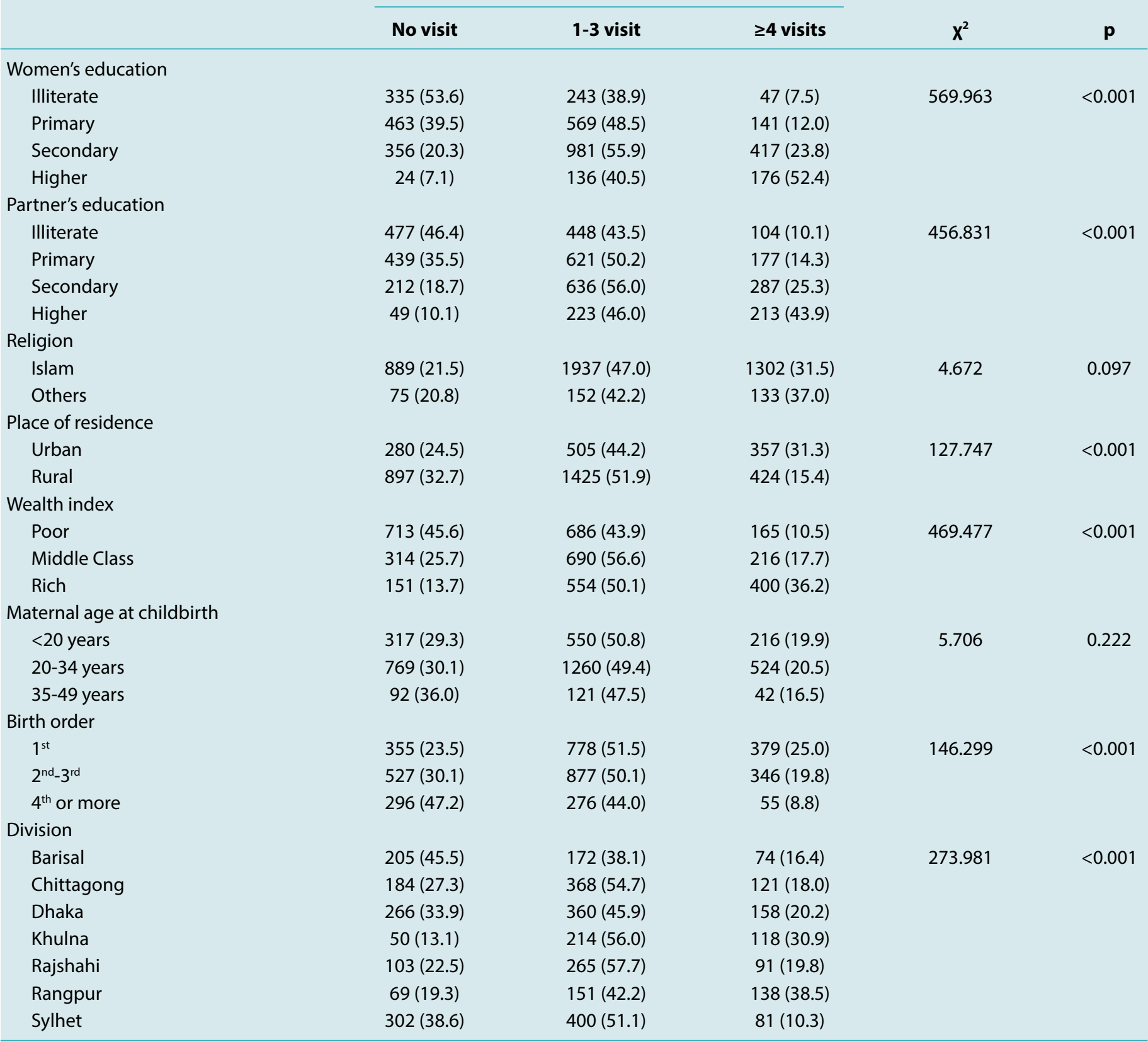

\section{Explanatory variables}

\section{ANC Visit}

ANC: Antenatal care.

Data are presented as $\mathrm{n}(\%)$.

Chi-square test. 
for women from urban and rural areas, respectively. The number of ANC visits increased for higher-income households. Rich women had a relatively more percentage of at least four visits in comparison to poor women. Moreover, mothers availing ANC visits decreased if the number of their children increased. Lastly, the divisions within the country sharply influenced ANC services. Results obtained from bivariate analyses are displayed in Table 1.
The chance of ANC attendance was higher among educated women from the table. To be precise, primary educated women were $(1.344-1)^{*} 100=34.4 \%$ more likely to receive $1-3$ ANC visits and $(1.306-1) * 100=30.6 \%$ more likely to receive at least four ANC visits than illiterate women. The likelihood of receiving ANC services was also higher for secondary $(40.7 \%$ for $1-3$ visit and $45.4 \%$ for $\geq 4$ visits) and highly (49.0\% for $1-3$ visit and $44.1 \%$ for $\geq 4$ visits) educated women. For partner's

Table 2. Estimation of adjusted effects for the selected explanatory variables with the attendance of antenatal care services

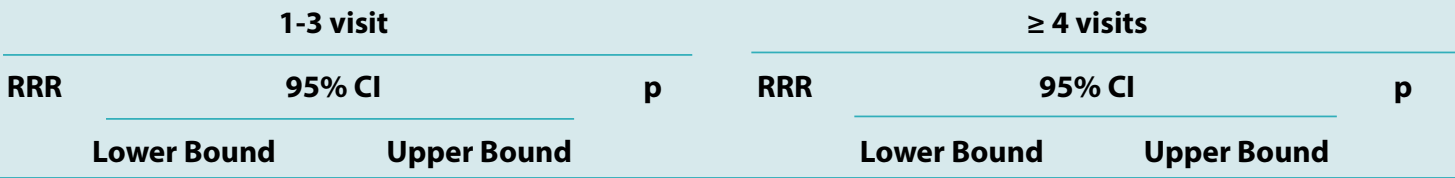

Women's education

Illiterate (ref)

Primary

Secondary

Higher secondary

Partner's education

Illiterate (ref)

Primary

Secondary

Higher Secondary

Religion

Islam (ref)

Others

Place of residence

Urban (ref)

Rural

Wealth index

Poor (ref)

Middle

Rich

1.344

1.253

$1.407 \quad 1.606$

1.490

1.388

1.132

0.905

1.352

1.100

1.478

1.255

0.983

0.732

0.711

0.555

1.209

1.114

1.506

1.307

Maternal age at childbirth $<20$ (ref)

20-34

35-49

$0.764 \quad 0.610$

0.508

0.339

Birth order

$1^{\text {st }}$ (ref)

$2^{\text {nd }}-3^{\text {rd }}$

$4^{\text {th }}$ or more

0.454

0.288

0.301

0.214

Division

Barisal (ref)

Chittagong

Dhaka

Khulna

Rajshahi

Rangpur

Sylhet

$\begin{array}{ll}1.176 & 0.890 \\ 0.623 & 0.493 \\ 0.457 & 0.388 \\ 0.853 & 0.666 \\ 0.579 & 0.484 \\ 1.362 & 0.977\end{array}$

1.747

2.207

2.112

$<0.001$

$<0.001$

$<0.001$

1.306

1.454

1.441

1.203
1.542

1.232

$0.100 \quad 1.041$

$<0.001$

0.846

$<0.001$

0.803

0.837

0.676

0.590

0.100

0.886

0.689

1.139

0.100

0.891

$<0.001$

0.787

.

1.644

1.970

$<0.001$

$<0.001$

1.304

1.465

1.071

1.188

1.582

1.787

$<0.001$

$<0.001$

0.970

0.010

0.890

0.770

$<0.001$

0.671

0.733

0.454

1.074

0.984

0.100

0.010

0.654

0.450

$<0.001$

0.410

$<0.001$

0.346

0.955

0.288

1.362

0.100

0.487

$<0.001$

AIC: Akaike information criterion; Cl: Confidence interval; RRR: Relative risk ratio.

Multinomial logistic regression model. 
education, women with secondary and highly educated partners had $(1.352-1)^{*} 100=35.2 \%$ and $(1.490-1) * 100=49.0 \%$ more chance to receive 1-3 ANC visits when compared to women with illiterate partners, respectively. Relative to poor, middle (20.9\% for $1-3$ visit and $30.4 \%$ for $\geq 4$ visits) and rich (50.6\% for $1-3$ visit and $97.0 \%$ for $\geq 4$ visits) women were more likely to attend ANC services. The chance of adopting ANC services was also lower for rural (28.9\% for $1-3$ visit and $21.3 \%$ for $\geq 4$ visits) women compared to their urban counterparts. Moreover, the chance of ANC attendance decreased with advancing maternal age and increment in birth order. Among the divisions, the chance of adopting ANC services was lower in Dhaka (37.7\% for $1-3$ visit and $19.9 \%$ for $\geq 4$ visits), Khulna ( $53.8 \%$ for $1-3$ visit and $43.2 \%$ for $\geq 4$ visits), and Rangpur (42.1\% for $1-3$ visit and $61.2 \%$ for $\geq 4$ visits) as compared to Barisal. The adjusted effects of selected explanatory variables were obtained by fitting MLR in Table 2.

From the table, the odds of shifting in a better state (1-3 visits and $\geq 4$ visits) of ANC visit was (1.252-1)*100 $=25.2 \%$ higher for primary educated women compared to uneducated women. These odds were $(1.333-1) * 100=33.3 \%$ and $(1.411-1)^{*} 100=41.1 \%$ higher or secondary and higher secondary educated women, respectively. Partner's education also showed similar strength of association. To be specific, the odds of shifting in a better state of ANC visit were $(1.166-1)^{*} 100=16.6 \%$ higher for a secondary educated partner and $(1.379-1) * 100=37.9 \%$ higher for a highly educated partner than an uneducated partner. Compared to women from poor income families, the odds of switching from a worse state to a better state of ANC visit was $(1.410-1)^{*} 100=41.0 \%$ and $(1.523-1)^{*} 100=52.3 \%$ higher for women from middle and rich income families, respectively. The rate of receiving ANC services during pregnancy was significantly lower for rural $(1-0.680) * 100=32.0 \%$ women as compared to urban women. Besides, the odds of receiving ANC services decreased significantly for older mothers and advancing birth order. Among divisions, the chance of receiving ANC services was relatively lower in Dhaka (24.5\%), Khulna (45.8\%), and Rangpur (55.6\%) in comparison to Barisal. The estimated effects of selected covariates obtained from the POM model are displayed in Table 3.

\section{DISCUSSION}

The study evaluated a nationally representative survey of Bangladesh and identified the risk factors related to ANC visits. According to bivariate analysis, women's education, partner's education, place of residence, wealth index, maternal age at birth, birth order, religion, and region of residence were significantly correlated with access to ANC services. Besides, the adjusted effects were estimated using
MLR and POM. The MLR model provided a lower AIC value between these two equipped models. Therefore, the MLR model better-suited the data. However, results obtained from MLR and POM were almost similar, although the MRL model had a smaller AIC value.

Results of the MLR model revealed that better-educated women were more likely to utilize ANC services compared to illiterate women. The result is plausible as education is feasible means of gathering knowledge and better-educated women have better knowledge regarding the importance of ANC services during pregnancy. This result is also supported by previous studies. ${ }^{[21-25]}$ Partner's education plays a similar role, which also coincides with other studies. ${ }^{[21,24]}$ The findings of this study also suggested that older mothers tended to have higher utilization of ANC, which is similar to the past study as well. ${ }^{[24,26,27]}$

Place of residence and region of residence were also found significant to the response variable. The probable cause may be the regional variation. Rural residents are poor and not well educated than their urban counterparts that result in a lower likelihood of receiving ANC services. This regional variation is also related to several socioeconomic factors, traditions and cultures of different regions. Moreover, various psychosocial features are associated with a lower likelihood of receiving ANC services among rural women and women who come from several regions of Bangladesh.

Wealth index was positively associated in the study with receiving ANC services. The illustration of this finding is intelligible. Limited livelihood is directly associated with poverty, which is affecting a lower chance of receiving ANC services among women living in a poor setting. Costly healthcare services are another reason for the lower utilization of ANC services, especially in these extreme poverty regions. ${ }^{[28]}$ Therefore, financing in the health sector through insurance or different organizations may reduce these obstacles. The study suggested that birth order was also negatively related to receiving ANC services. Fear of miscarriage and excitement of being pregnant for the first time is resulting in a higher likelihood of receiving ANC during pregnancy and this aligns with previous studies. ${ }^{[18,29,30]}$ Previously, similar research was performed in Turkey with a sample of 446 females ${ }^{[31]}$ However, the study did not represent the whole population of Turkey due to its small sample size. Therefore, the study recommends the implementation of this sort of research and interventions (according to explanatory variables) in Turkey since it is a significant topic for healthcare professionals regardless of any socioeconomic context. 
Table 3. Estimation of adjusted effects for the selected explanatory variables with the attendance of antenatal care services

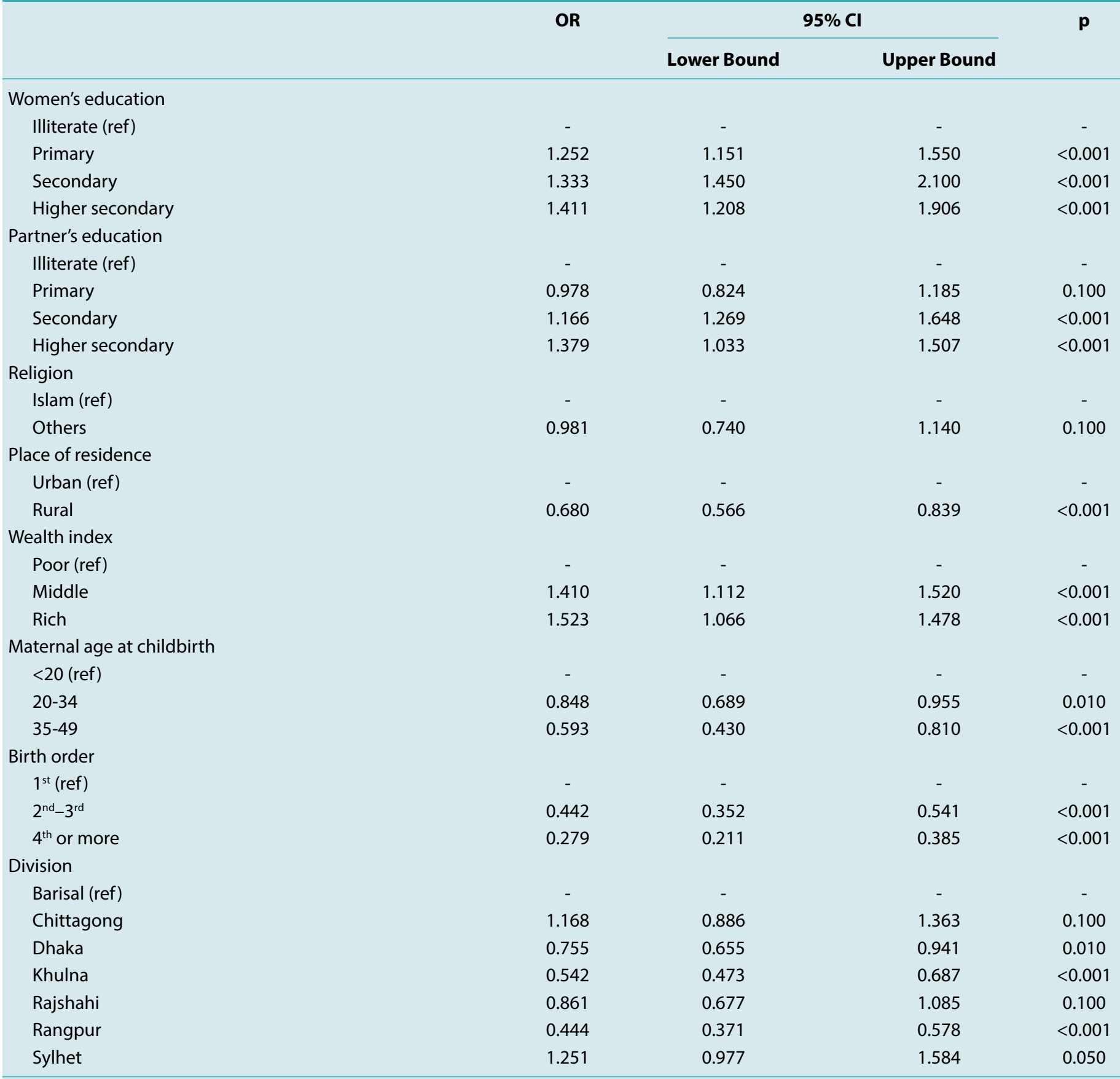

Model AIC value $=8324.07$

AIC: Akaike information criterion; Cl: Confidence interval; OR: Odds ratio.

Proportional odds model.

This study has several limitations. First, we evaluated a cross-sectional retrospective dataset. Second, the failure to update important statistics in time in Bangladesh can lead to errors when reporting a mother during childbirth. Third, not all potential risk factors (mass media exposure, distance from the healthcare center to your home, etc.) cannot be included. However, examining follow-up analysis can help you achieve a better overview. Despite these shortcomings, the main strength of this study is that it surveyed representative national surveys that collected data using appropriate probability sampling techniques. 


\section{CONCLUSION}

ANC services play a vital role in reducing maternal mortality and morbidity. Financial obstacles and lack of knowledge about the importance of ANC services are the vital barriers. This study revealed that lower educated and poor women were less likely to receive ANC services. The barrier connected to education and financial support should be addressed carefully and can be reduced by increasing women's education and financing the health sector through insurance or organizations. This study also suggested strengthening maternal education especially in rural areas of Bangladesh. Special focus should be needed in Dhaka, Khulna, Rangpur, and Sylhet divisions due to lower utilization regions of ANC services. Finally, the use of ANC services during the pregnancy cycle undoubtedly would reduce the risk of child malnutrition and mortality, maternal mortality and morbidity.

\section{Disclosures}

Acknowledgments: We would like to thank the National Institute of Population Research and Training and MEASURE DHS for giving us permission to use BDHS 2014 data for analysis.

Availability of Data and Materials: The study analyzed the secondary dataset of BDHS 2014, which is freely available on the following website: http://dhsprogram.com/data/available-datasets.cfm.

Peer-review: Externally peer-reviewed.

Conflict of Interest: None declared.

Ethics Committee Approval: This study was performed with the approval of the Institutional Review Board Findings Form ICF IRB FWA00000846 (Approval date: "March 11, 2015, Approval number: 132989.0.000).

Authorship Contributions: Concept - A.T., T.S., N.N.P., M.A.M.; Design -A.T., T.S.; Supervision - A.T.; Materials -A.T., T.S., N.N.P., M.A.M.; Data collection \&/or processing -T.S., N.N.P., M.A.M, H.R.H.; Analysis and/or interpretation -T.S., N.N.P., M.A.M, H.R.H, B.A.; Literature search -A.T., T.S., N.N.P., M.A.M., H.R.H; Writing -A.T., T.S., N.N.P., M.A.M., H.R.H.; Critical review -A.T., T.S., N.N.P., M.A.M., H.R.H, B.A.

\section{REFERENCES}

1. WHO. World Health Report 2005. Make every mother and child count. Available at: http://www.who.int/whr/2005/ whr2005_en.pdf. Accessed Feb 18, 2021.

2. UNICEF. Progress for Children: A report card on maternal mortality, no. 7 Division of Policy and Planning, 2008. http://www. unicef.org/childsurvival/files/Progress_for_Children-No._7_ Lo-Res_082008. Accessed Feb 18, 2021.

3. Kvale G, Olsen BE, Hinderkar SG, Ulstein M, Bergsjø P. Maternal deaths in developing countries: a preventable tragedy. Norsk
Epidemiol 2005;15(2):141-9. [CrossRef]

4. Adam T, Lim S, Mehta S, Bhutta ZA, Fogstad H, Mathai M, et al. Cost effectiveness analysis of strategies for maternal and neonatal health in developing countries. Br Med J 2005;331(7525):1107. [CrossRef]

5. MCcaw-Binns A, Lagrenade J, Ashley D. Under-users of antenatal care: a comparison of non-attenders and late attenders for antenatal care with early attenders. Soc Sci Med 2007;40(7):1003-12. [CrossRef]

6. Abosse Z, Woldie M, Ololo S. Factors influencing antenatal care service utilization in hadiya zone. Ethiop J Health Sci 2010;20(2):75-82. [CrossRef]

7. USAID 2007: Focused antenatal care: providing integrated, individualized care during pregnancy. Available at: http://www. accesstohealth.org/toolres/pdfs/ACCESStechbrief_FANC.pdf.

8. WHO, UNICEF. Antenatal care in developing countries: promises, achievement and Missed opportunities: an analysis of trends, levels, and differentials. Available at: https://www. who.int/reproductivehealth/publications/maternal_perinatal_health/9241590947/en/. Accessed Feb 18, 2021.

9. Finlayson $K$, Downe S. Why do women not use antenatal services in low-and-middle-income countries? A meta-synthesis of qualitative studies. PLoS Med 2013;10(1):e1001373. [CrossRef]

10. WHO. New guidelines on antenatal care for a positive pregnancy experience. Sexual Reprod Health 2016.

11. Pell C, Meñaca A, Were F, Afrah NA, Chatio S, Manda-Taylor L, et al. Factors affecting antenatal care attendance: results from qualitative studies in Ghana, Kenya and Malawi. PLoS One 2013;8(1):e53747. [CrossRef]

12. Rahman MH, Mosley WH, Ahmed S, Akhter HH. Does service accessibility reduce socioeconomic differentials in maternity care seeking? Evidence from rural Bangladesh. J Biosoc Sci 2008;40(1):19-33. [CrossRef]

13. Anwar I, Kalim N, Koblinsky M. Quality of obstetric care in public-sector facilities and constraints to implementing emergency obstetric care services: evidence from high and low-performing districts of Bangladesh. J Health Popul Nutr 2009;27(2):139-55. [CrossRef]

14. Bauserman M, Lokangaka A, Thorsten V, Tshefu A, Goudar SS, Esamai F, et al. Risk factors for maternal death and trends in maternal mortality in low- and middle-income countries: a prospective longitudinal cohort analysis. Reprod Health 2015;12(Suppl 2):S5. [CrossRef]

15. Carroli G, Rooney C, Villar J. How effective is antenatal care in preventing maternal mortality and serious morbidity? An overview of the evidence. Paediatr Perinat Epidemiol 2001;15 (Suppl 1):1-42. [CrossRef]

16. McDonagh M. Is antenatal care effective in reducing maternal morbidity and mortality? Health Policy Plan 1996;11(1):1-15.

17. National Institute of Population Research and Training (NIPORT), Mitra and Associates. Bangladesh Demographic and 
Health Survey 2014. Available at: https://dhsprogram.com/ pubs/pdf/FR311/FR311.pdf. Accessed Feb 18, 2021.

18. Kabir MR. Effect of women education on the utilization of antenatal care (ANC) services in Bangladesh: A multivariate analysis of socioeconomic and demographic. Academia J Educ Res 2016;4(6):95-103.

19. Mohammad KA, Zahura FT, Rahman MM. Importance of maternal education on antenatal care visits in Bangladesh. Bangladesh J Sci Res 2017;30(1-2):23-33. [CrossRef]

20. Agresti A. Analysis of ordinal categorical data. New York: John Wiley \& Sons; 2010. [CrossRef]

21. Tey NP, Lai SL. Correlates of and barriers to the utilization of health services for delivery in South Asia and Sub-Saharan Africa. Sci World J 2013;2013:1-11. [CrossRef]

22. Navaneetham K, Dharmalingam A. Utilization of maternal health care services in southern India. Soc Sci Med 2002;55(10):1849-69. [CrossRef]

23. Raghupathy S. Education and the use of maternal health care in Thailand. Soc Sci Med 1996;43(4):459-71. [CrossRef]

24. Maraga S, Namosha E, Gouda H, Vallely L, Rare L, Phuanukoonnon S. Sociodemographic factors associated with maternal health care utilization in Wosera, East Sepik Province, Papua New Guinea. PNG Med J 2011;54(3-4):154-63.

25. Prusty RK, Buoy S, Kumar P, Pradhan MR. Factors associated with utilization of antenatal care services in Cambodia. J Public Health 2015;23(5):297-310. [CrossRef]

26. Kamal SM, Hassan $\mathrm{CH}$, Islam MN. Factors associated with the timing of antenatal care seeking in Bangladesh. Asia Pac J Public Health 2015;27(2):NP1467-80. [CrossRef]

27. Regassa N. Antenatal and postnatal care service utilization in southern Ethiopia: a population-based study. Afr Health Sci 2011;11(3):390-7.

28. Ali A, Osman M, Abbaker AO, Adam I. Use of antenatal care services in Kassala, eastern Sudan. BMC Pregnancy Childbirth 2010;10(1):67. [CrossRef]

29. Agha S, Carton TW. Determinants of institutional delivery in rural Jhang, Pakistan. Int J Equity Health 2011;10:31. [CrossRef]

30. Magadi MA. Maternal and child health among the urban poor in Nairobi, Kenya. African Popul Stud 2004;19:172-90.

31. Erci B. Barriers to utilization of prenatal care services in Turkey. J Nurs Scholarsh 2003;35(3):269-73. [CrossRef] 